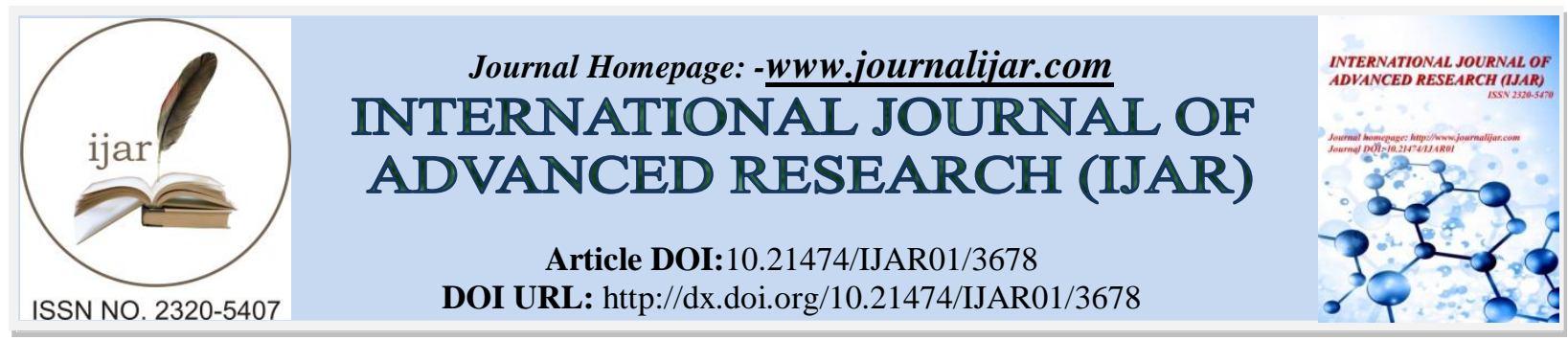

RESEARCH ARTICLE

\title{
CORRELATION OF ALT/AST RATIO WITH INSULIN RESISTANCE IN METABOLIC SYNDROME.
}

\author{
Ramya SG ${ }^{1}$, Rajinder K Dhamija ${ }^{2}$, Anil Gurtoo ${ }^{3}$ and Ritu Singh ${ }^{4}$. \\ 1. Ramya SG-Junior Resident, Department of General Medicine, Lady Hardinge Medical College, Newdelhi. \\ 2. Rajinder K Dhamija - Professor, Department of General Medicine, Lady Hardinge Medical College, Newdelhi. \\ 3. Anil Gurtoo -Professor, Department of General Medicine, Lady Hardinge Medical College, Newdelhi. \\ 4. Ritu Singh - Professor, Department of Biochemistry, Lady Hardinge Medical College, Newdelhi.
}

\section{Manuscript Info}

Manuscript History

Received: 12 January 2017

Final Accepted: 05 February 2017

Published: March 2017

Key words:-

ALT, HOMA-IR, Metabolic syndrome

\begin{abstract}
Background:In recent years, non-alcoholic fatty liver disease is considered as a novel component of insulin resistance and metabolic syndrome, which is found to be associated with mild elevation of liver enzymes.

Aim of the Study:The aim of this study was to correlate the ALT/AST ratio with insulin resistance calculated by HOMA-IR (Homeostatic model assessment-Insulin resistance) method among patients found to have metabolic syndrome.

Design and Methodology: This was an observational cross sectional study which included 60 subjects of metabolic syndrome defined by International Diabetes Federation criteria. The relevant clinical examination and basic investigations were done. Fasting insulin levels were analysed by chemiluminescence method. Liver function tests were done by fully automated AnalyzerSynchron CX-9 using Roche kits, calibrators and Randox control Sera. Data was processed and analyzed by SPSS version 17.0 software. The Correlation between ALT/AST ratio and HOMA-IR was assessed using The Pearson correlation test.

Results The ALT/AST Ratio was found to have positive correlation with HOMA-IR (Pearson's correlation coefficient is +0.742 and the significance was $<0.001)$. This study shows that ALT/AST ratio can be used as screening tool of insulin resistance in the community.
\end{abstract}

Copy Right, IJAR, 2017,. All rights reserved.

\section{Introduction:-}

The aminotransferases (transaminases) are sensitive indicators of liver cell injury.They include the aspartate aminotransferase (AST) and the alanine aminotransferase (ALT). AST is found in the liver, cardiac muscle, skeletal muscle, kidneys, brain, pancreas, lungs, leukocytes, and erythrocytes in decreasing order of concentration. ALT is found primarily in the liver and is therefore a more specific indicator of liver injury [1].

The aminotransferases are normally present in the serum in low concentrations. These enzymes are released into the blood in greater amounts when there is damage to the liver cell membrane resulting in increased permeability. The normal range for aminotransferases varies widely among laboratories, but generally ranges from 10-40 U/L [1].

Corresponding Author:-Ramya SG.

Address:-Ramya SG-Junior Resident, Department of General Medicine, Lady Hardinge Medical College, Newdelhi. 
The liver plays an important role in maintaining normal glucose concentrations during fasting as well as postprandially. A number of recent studies have suggested that abnormal hepatocellular function is associated with obesity, insulin resistance, and type 2 diabetes [2], [3].

The metabolic syndrome is a condition characterized by a cluster of features including glucose intolerance, insulin resistance, abdominal obesity, atherogenicdyslipidemia, elevated blood pressure, a proinflammatory and a prothrombotic state [4]. It increases the mortality and morbidity due to cardiovascular diseases significantly. Insulin resistance is the key feature of metabolic syndrome. It means greater amount of insulin are required to elicit normal biologic response at a cellular organ or whole body level [4].

In recent years, nonalcoholic fatty liver disease (NAFLD), as a novel component of insulin resistance and metabolic syndrome (MS), has drawn the attention of many researchers. NAFLD encompasses a wide spectrum of liver diseases ranging from simple benign steatosis to steatohepatitis, fibrosis and cirrhosis. This condition which is associated with long-standing elevations in liver enzymes is related to higher risk of adverse cardiovascular events, oxidative stress, endothelial dysfunction, and MS [5]. Although not all patients with NAFLD are obese, obesity is considered the most important risk factor [5], [6].Typically, liver biopsy is the ultimate test for diagnosis and delineation of the extent. It is usually not done except for situations of conflicting diagnosis. Liver ultrasonography although not sensitive enough to differentiate simple steatosis from more advanced hepatic involvement, is widely used [7].Recently ALT/AST ratio has attracted great interest as potential novel marker of insulin resistance.

\section{Aim ofthe Study:-}

The aim of this study was to correlate the ALT/AST ratio with insulin resistance calculated by HOMA-IR (Homeostatic model assessment-Insulin resistance) method in metabolic syndrome patients.

\section{Materials and Methods:-}

This was an observational cross sectional study conducted among 60 patients of age $\geq 18$ years with metabolic syndrome. This study was approved by the Ethical committee of the institution.

\section{Inclusion Criteria:-}

The study population included the adult patients of age $\geq 18$ years who were diagnosed to have Metabolic syndrome based on following International Diabetes Federation criteria.

Waist circumference $\geq 90 \mathrm{~cm}$ in men and $\geq 80 \mathrm{~cm}$ in women with two or more of the following:-

Fasting triglyceride $\geq 150 \mathrm{mg} / \mathrm{dl}$ or specific medications.

HDL cholestrol $<40 \mathrm{mg} / \mathrm{dl}$ and $<50 \mathrm{mg} / \mathrm{dl}$ for men and women respectively or specific medications.

Blood pressure $\geq 130 \mathrm{~mm}$ systolic or $\geq 85 \mathrm{~mm}$ diastolic or previously diagnosed hypertensive or specific medications.

Fasting blood glucose $\geq 100 \mathrm{mg} / \mathrm{dl}$ or previously diagnosed type 2 Diabetes mellitus.

\section{Exclusion Criteria:-}

The following cases were excluded from the study:-

History of alcohol intake.

History of liver disease due to autoimmune, viral, genetic, drug induced causes.

Severe cardiac/renal/hepatic dysfunction.

Patient on insulin therapy for treatment of diabetes mellitus.

\section{Methodology:-}

A detailed history and examination of the study population were noted and anthropometric measurements, including height, weight, BMI, waist circumference and hip circumference were noted and the waist hip ratio was calculated(WHR). $10 \mathrm{ml}$ of fasting venous blood sample was taken with aseptic precautions and distributed in EDTA, plain and fluoride containers. EDTA sample was used for complete blood count, fluoride vial sample for blood glucose and serum for estimation of Liver function tests, Kidney function tests and Lipid profile. Fasting insulin level in a plain test tube was analysed by chemiluminescence method. HBsAg and Hepatitis C were tested by Elisa method. Liver function tests, kidney function test and lipid profile were checked using fully automated AnalyzerSynchron CX-9. Normal ALT and AST levels were considered to be 10-40 IU /L. 
Insulin resistance is calculated by following formula using homeostasis model assessment of insulin resistance,

HOMA-IR $=\underline{\text { fasting insulin }(\mu \mathrm{U} / \mathrm{ml}) \times \text { fasting glucose }(\mathrm{mmol} / \mathrm{l})}$

Statistical analysis ofthestudy:-

Data was processed and analysed by SPSS version 17.0 software. Sample statistics i.e, mean, median and standard deviation were calculated for quantitative data. Chi-square test and difference of proportion were applied for qualitative analysis. Mean and standard deviation were compared by student $t$ test. Mann Whitney's test and Kruskal Wallis test were used for non-normally distributed data. The Correlation between ALT/AST ratio and HOMA-IR was assessed using the Pearson correlation. Statistical significance was set at $\mathrm{p} \leq 0.05$.

\section{Results:-}

$68 \%$ of patients were from the group of age 50-69 years and $8 \%$ of patients were from the group of age $\geq 70$ years and $23 \%$ from the group of age $30-49$ years (table 1). The mean age is found to be $57.05 \pm 8.83$ years. The study population ranged from 36 years to 74 years. There were 30 males and 30 females. There was no statistically significant difference between the age distribution of males and females $(\mathrm{p}=0.806)$.

Patients who were both Diabetic and hypertensive patients (43.33\%) were more in number compared to isolated diabetes(26.67\%) and isolated hypertension(28.33\%). The Blood pressure did not show any statistically significant difference among sex groups and different age groups but diastolic BP was significantly higher with people having higher BMI.The mean BMI was $29.44 \pm 2.83 \mathrm{~kg} / \mathrm{m} 2$. Majority (93.33\%) of study group were with BMI $>25 \mathrm{~kg} / \mathrm{m} 2$ and females were found to have higher mean BMI. $68.3 \%$ patients among the study group had WHR $\geq 0.95$. The mean fasting Blood sugar among study group was $170.12 \pm 36.37 \mathrm{mg} / \mathrm{dl}$.

The mean ALT level among the study group is $51.45 \pm 9.12 \mathrm{IU} / \mathrm{L}$. The mean AST level among the study group is $44.18 \pm 6.19 \mathrm{IU} / \mathrm{L}$. The Study group is divided based on normal cut off as $40 \mathrm{IU} / \mathrm{L}$ for both the enzymes and it was found that 56 patients $(93.33 \%$ ) were found to have ALT levels $\geq 40 \mathrm{IU} / \mathrm{L}$ and 36 patients $(60 \%)$ were found to have AST $\geq 40 \mathrm{IU} / \mathrm{L}$ (table 2). The mean ALT/AST Ratio among the study group is $1.173 \pm 0.186$. The ALT/AST Ratio among the study group ranged from 0.69 to $1.60 .83 .3 \%$ of patients were in the group ALT/AST RATIO $\geq 1$ (figure $1)$.

The mean HOMA-IR level among the study group is $4.14 \pm 2.27$.The HOMA-IR among the study group ranged from 1.23 to 11.28. The definite cut off of HOMA-IR to identify insulin resistance is not defined yet as the people in different areas of world also differs in their ethnicity and clinical background. On considering patients whose HOMA IR $\geq 2$ as insulin resistant and $\mathrm{HOMA}<2$ as non insulin resistant, it was found that about $81.7 \%$ patients among the study group were insulin resistant.

The ALT/AST Ratio was correlated to HOMA-IR among the study group using Pearson Correlation test and it was found that Pearson's Correlation coefficient is +0.742 and the significance( 2 tailed) was $<0.001$ (Figure 2 ). Hence the correlation was found to be statistically significant. The correlation between ALT enzyme levels and HOMA-IR was also found to be statistically significant $(\mathrm{r}=+0.58$ and $\mathrm{p}<0.001$.

There was positive correlation between ALT/AST Ratio with fasting Blood Sugar and fasting insulin levels with significance ( 2 tailed ) value $<0.001$. The ROC curve of ALT/AST Ratio is calculated in relation to HOMA-IR value as gold standard test (figure 3). Among the study group it was found that about 49 patients had HOMA-IR $>2$ where as 11 patients had HOMA-IR<2. The area under the curve is 0.862 and it was found that the cut off of HOMA-IR value-1.06 has the sensitivity of $93.9 \%$ and the specificity of $81.8 \%$ but the cut off of 1.07 has the sensitivity of 91.8 $\%$ and specificity of $81.8 \%$. Since ALT/AST Ratio is easy to use as a screening tool of insulin resistance the cut off of 1.06 is considered better to achieve better sensitivity. 
Table 1:- Age and Sex distribution of study group.

\begin{tabular}{|c|c|c|c|}
\hline Sex & Age 30-49 years & Age 50-69 years & Age $\geq 70$ years \\
\hline Male & $6(20 \%)$ & $21(70 \%)$ & $3(10 \%)$ \\
\hline Female & $8(26.66 \%)$ & $20(66.66 \%)$ & $2(6.67 \%)$ \\
\hline Total & $14(23 \%)$ & $41(68 \%)$ & $5(8 \%)$ \\
\hline
\end{tabular}

Table 2:- Frequency distribution of ALT, AST and HOMA-IR.

\begin{tabular}{|c|c|c|}
\hline Parameter & Frequency & Percentage \\
\hline ALT $<40$ IU/L & 4 & $6.7 \%$ \\
\hline ALT $\geq 40 \mathrm{IU} / \mathrm{L}$ & 56 & $43.3 \%$ \\
\hline $\mathrm{AST}<40$ & 24 & $60 \%$ \\
\hline $\mathrm{AST} \geq 40$ & 36 & $16.7 \%$ \\
\hline ALT/AST<1 & 10 & $83.3 \%$ \\
\hline ALT/AST $\geq 1$ & 50 & $81.7 \%$ \\
\hline HOMA-IR $\geq 2$ & 49 & $18.3 \%$ \\
\hline HOMA-IR $<2$ & 11 & \\
\hline
\end{tabular}

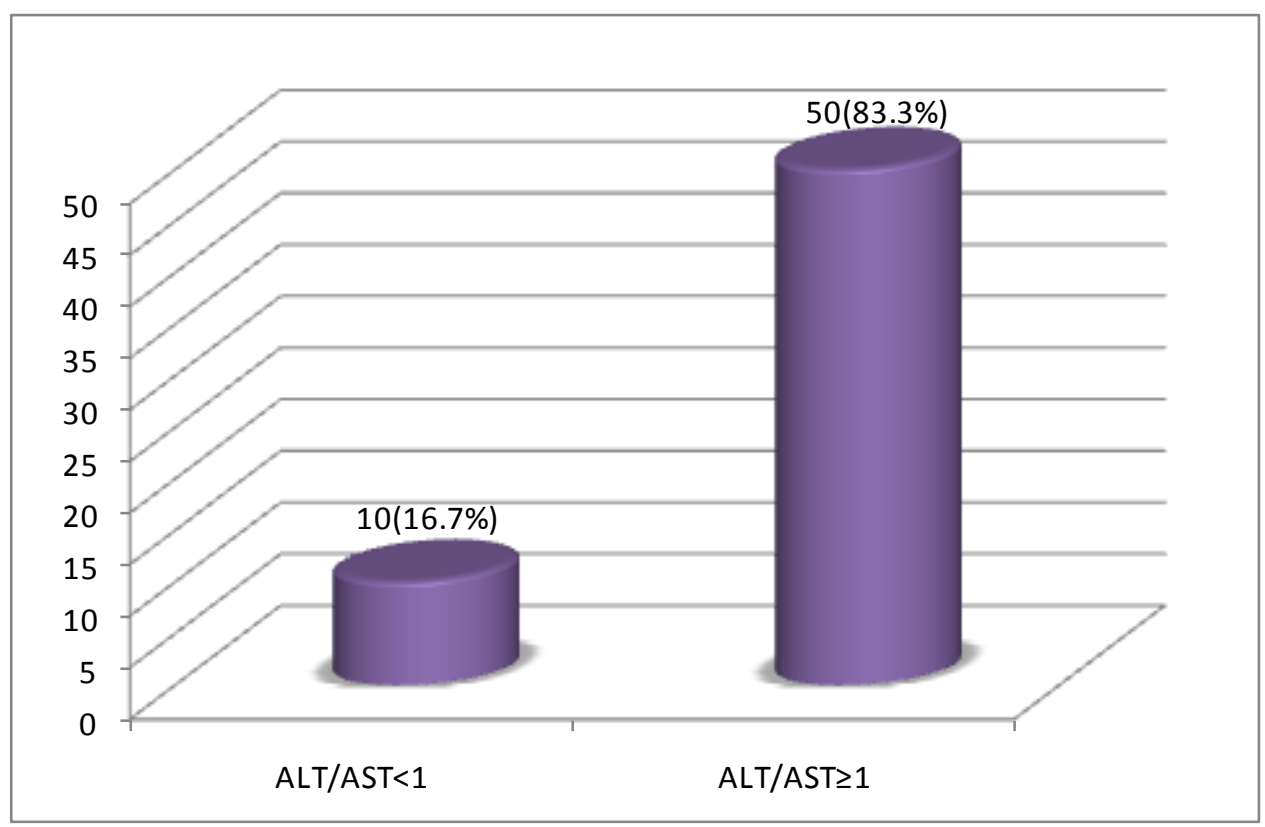

Figure 1:- Distribution of ALT/AST Ratio among study group. 


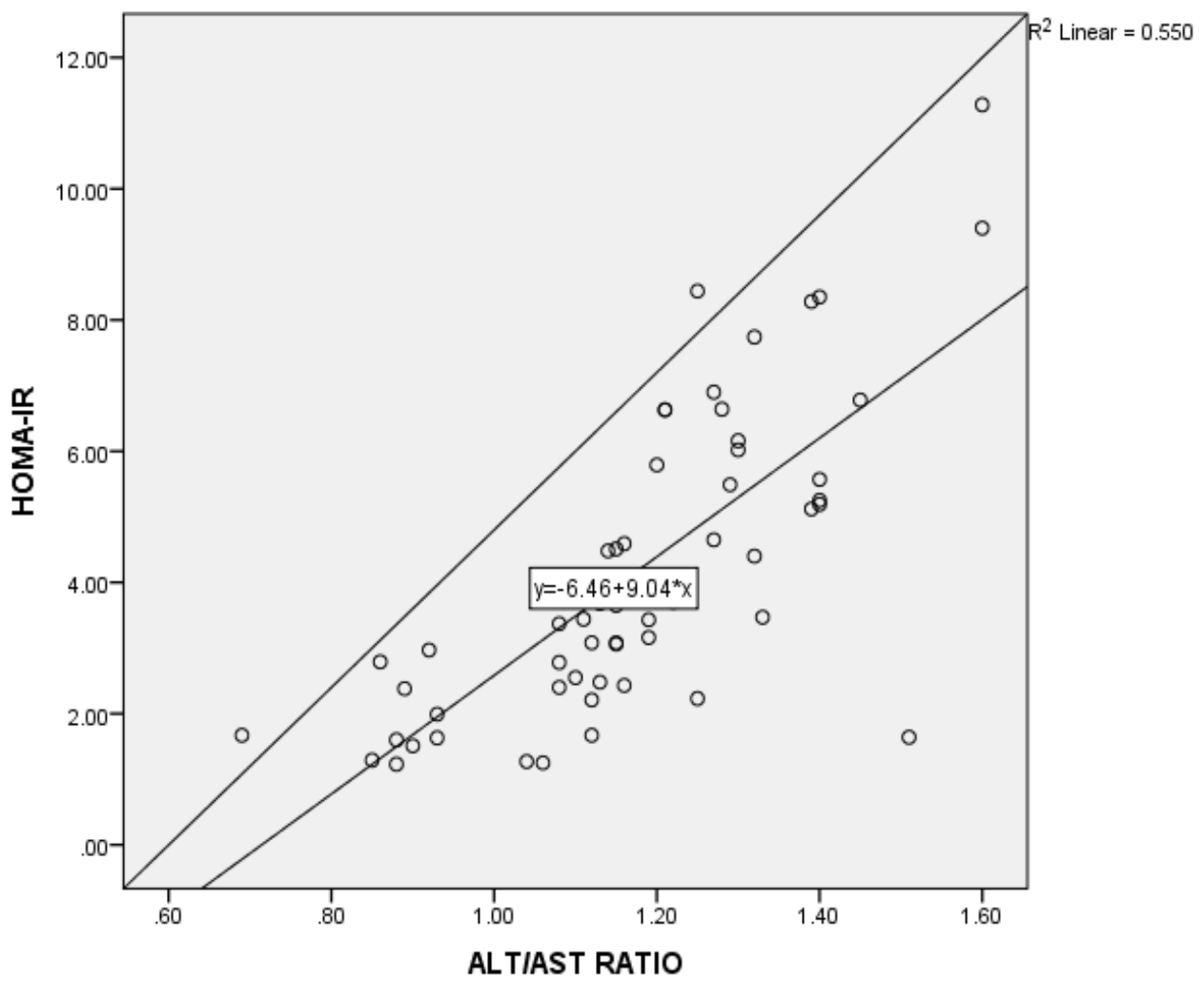

Figure 2:- Scatter Plot showing correlation of ALT/AST ratio toHOMA-IR.

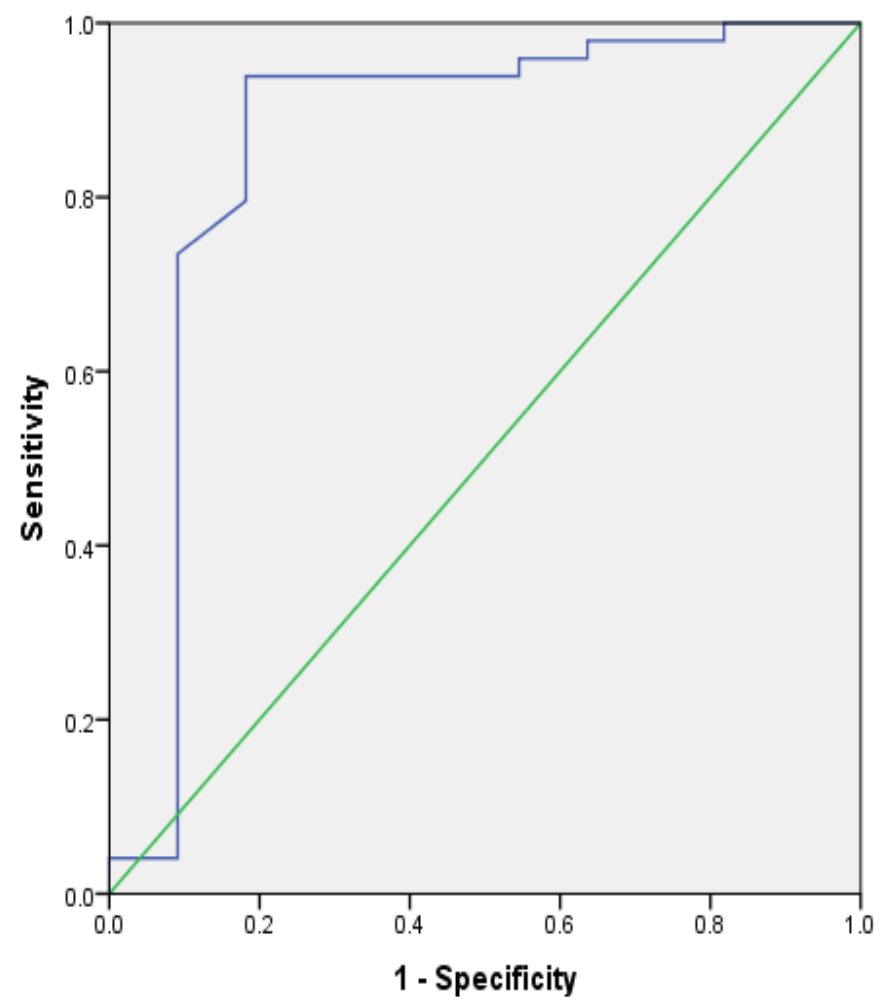

Figure 3:- ROC curve of ALT/AST ratio in relation to HOMA-IR. 


\section{Discussion:-}

The mean age of this study population was found to be 57.05 years which is similar to study in Eastern India by Prasad et al [8] which showed that prevalence of metabolic syndrome increases with age and maximum number of patients were from age 50-59 years. Another study by Kumar et al [9] in Warangal, Andhra pradesh also showed maximum prevalence was found in age group 51 -60 years with a mean of 52.8 \pm 11.6 years. Deepa et al [10] conducted a study conducted in Chennai among metabolic syndrome patients defined by IDF criteria showed mean age to be $44 \pm 11$ years. Tan et al [11] studied metabolic syndrome among Asians which also showed increasing prevalence of metabolic syndrome and maximum prevalence was among age group 60-69 years (50.8\%). Kanjilal et al [12] conducted a study among metabolic syndrome patients from Bangalore and Mumbai also showed maximum prevalence among the age group 50-59 years.

26 patients among our study group (43.33\%) were suffering from both diabetes and hypertension and 16 patients were only diabetic and 17 patients were only hypertensive. There was one patient with impaired glucose tolerance. This shows that patients with both Diabetic and hypertensive patients were more in number compared to isolated diabetes and isolated hypertension. But Prasad et al [8] it was shown that hypertension is the most common abnormality observed among metabolic syndrome patients with about $89.2 \%$ prevalence.

The Mean BMI among our study group was $29.44 \mathrm{~kg} / \mathrm{m} 2$ and $93.33 \%$ of study group had BMI $>25 \mathrm{~kg} / \mathrm{m} 2$. BMI was significantly higher among females. Prasad et al [8] showed that $67 \%$ of patients of metabolic syndrome were of BMI $>25 \mathrm{~kg} / \mathrm{m} 2$. Garg et al [13] in their study observed that mean BMI was $28.1 \pm 2.1 \mathrm{~kg} / \mathrm{m} 2$ similar to our study. Kumar et al [9] observed mean BMI of $25 \pm 4 \mathrm{~kg} / \mathrm{m} 2$ in their study. Bhat et al [14] observed a mean BMI of $25.01 \pm 3.24 \mathrm{~kg} / \mathrm{m} 2$ in their study. Indulekha et al [15] observed a mean BMI of $25.6 \pm 4.2 \mathrm{~kg} / \mathrm{m} 2$ in their study. Deepa et al [10] observed a mean BMI of $25.7 \pm 3.1 \mathrm{~kg} / \mathrm{m} 2$ in their study.

The mean Waist circumference /Hip circumference ratio was 0.95 and the standard deviation was 0.046 . It was found that $68.3 \%$ patients among the study group had WHR $\geq 0.95$. In a study from Qatar among metabolic syndrome patients it was found mean WHR was $0.89 \pm 0.09$. Deepa et al [10] observed a mean WHR of $0.94 \pm 0.08$. Garg et al [13] in their study observed a mean WHR of $1.18 \pm 0.1$ in males and $1.21 \pm 0.1$ in females.

In a study from Texas by Hanley et al [16] it was shown that ALT, ALP and CRP were found to have positive association with various components of metabolic syndrome .It was also found that there was negative association between AST/ALT ratio to components of metabolic syndrome. In another study among Mexican Children with Metabolic syndrome it was showed that higher prevalence of elevated ALT compared with those without the syndrome (30.9\% vs. 9.4\%), as well as higher mean serum ALT levels (42.1 IU/L, 95\%CI 33.4-50.7 vs. 23.9 IU/L, 95\%CI 21.0-26.8), and higher prevalence of an AST/ALT ratio <1 (47.3\% vs. 18.2\%) [17]. Among Korean adolescents, a study by Park et al showed positive association of ALT with components of metabolic syndrome [18]. AST/ALT ratio showed positive correlations with HDL-C and negative correlations with BMI, WC, TG, and HOMA-IR in a study from Taiwan [19]. ALT levels were significantly higher in subjects with MS compared to those without MS in another study from China [20].

In our study, ALT/AST Ratio was found to correlate with HOMA-IR value which is calculated using fasting blood sugar and fasting insulin levels among metabolic syndrome patients (pearson's correlation coefficient is +0.742 and the significance was $<0.001$ ). The HOMA-IR cut off of about 1.06 had the sensitivity of $93.9 \%$ and the specificity of $81.8 \%$. At this cut off, Positive Predictive Value was 95\% and Negative Predictive Value was $75 \%$. Positive Likelihood Ratio was 5.16 and Negative Likelihood Ratio was 0.07. Hanley et al [16] in their study "The Insulin Resistance Atherosclerosis Study" showed that elevated ALT was independently associated with insulin resistance by HOMA-IR method. In another study from China it was found that obesity and elevated ALT are significantly associated with insulin resistance by HOMA-IR method [21]. In a study among Japanese population [22] ALT/AST ratios were independently and significantly associated with HOMA-IR and the optimal cut-off point to identifying insulin resistance for these markers yielded ALT/AST ratio of $\geq 0.82$ in non-obese subjects and $\geq 1.02$ in overweight subjects to identify insulin resistance which is very similar to our study. In another study from China, both ALT and GGT concentrations were linearly correlated with HOMA-IR and independently associated with HOMA-IR [23]. To the contrary, in a study by Esteghamati et al, both Serum ALT and AST were significantly higher in patients with MS [24]. 


\section{Conclusion:-}

ALT/AST Ratio was found to have positive correlation with HOMA-IR. Fasting blood sugar and fasting insulin, ALT enzyme levels were also found to have positive correlation with HOMA-IR. The best cut off of ALT/AST Ratio to identify insulin resistance was 1.06 with sensitivity of $93.9 \%$ and the specificity of $81.8 \%$ among study group. $93.3 \%$ of patients with metabolic syndrome had raised ALT and $83.3 \%$ patients with metabolic syndrome had ALT/AST ratio $>1$.

\section{Acknowledgement:-}

We are thankful to management of Lady Hardinge medical college for permitting us to do this study and all the patients who participated in the same.

\section{References:-}

1. Pratt DS, Kaplan MM. Evaluation of abnormal liver enzyme results in asymptomatic patients.NEng1 J Med 2000; 342:1266-71.

2. Duckworth WC, Hamel FG, Peavy DE 1988 Hepatic metabolism of insulin. Am J Med85:71-76.

3. Michael MD, Kulkarni RN, Postic C, Previs SF, Shulman GI, Magnuson MA, Kahn CR: Loss of insulin signaling in hepatocytes leads to severe insulin resistance and progressive hepatic dysfunction. Mol Cell 6:8797, 2000.

4. Grundy S, Brewer B, Cleman J, Smith S, LenfantC.Definition of metabolic syndrome: Report of the National Heart,Lung,and the Blood institute/American Heart Association Conference on Scientific Issues Related to definition.Circulation 2004; 109:433-438.

5. Chitturi S, Abeygunasekera S, Farell GC, Holmes Walker J, Hui M, Fung C, Karim R. NASH and Insulin resistance: insulin hypersecretion and specific association with the insulin resistance syndrome.Hepatology 2002; 35: 373-379.

6. Marceau P, Biron S, Hould FS, Marceau S, Simard S, Thung SN, Kral JG. Liver pathology and Metabolic syndrome X in severe obesity. J ClinEndocrinolMetab1999; 84: 1513-1517.

7. S Banerjee, US Ghosh, S Dutta. Clinicopathological Profile of Hepatic Involvement in Type-2 Diabetes Mellitus and Its Significance.JAPI august 2008;vol 56:594-599.

8. Prasad DS, Kabir Z, Dash AK, Das BC. Prevalence and risk factors for metabolic syndrome in Asian Indians: A community study from urban Eastern India. J Cardiovasc Dis Res 2012; 3:204-11.

9. Kumar SV, Nagesh A, Leena M, Shravani G, Chandrasekar V. Incidence of metabolic syndrome and its characteristics of patients attending a diabetic outpatient clinic in a tertiary care hospital. J Nat ScBiol Med 2013; 4:57-62.

10. Deepa, M., Farooq, S., Datta, M., Deepa, R. and Mohan, V. 2007. Prevalence of metabolic syndrome using WHO, ATPIII and IDF definitions in Asian Indians: the Chennai Urban Rural Epidemiology Study (CURES34). Diabetes/metabolism research and reviews, 23 (2), pp.127-134.

11. Tan C., Ma, S., Wai, D., Chew, S. and Tai, E. 2004. Can we apply the National Cholesterol Education Program Adult Treatment Panel definition of the metabolic syndrome to Asians? Diabetes care, 27 (5), pp. 1182--1186.

12. Kanjilal S, Shanker J, Rao VS, Khadrinarasimhaih NB, Mukherjee M, Iyengar SS, et al. Prevalence and component analysis of metabolic syndrome: An Indian atherosclerosis research study perspective. Vasc Health Risk Manag. 2008; 4:189-97.

13. Garg MK, Dutta MK, Mahalle N. Study of beta-cell function (by HOMA model) in metabolic syndrome. Indian J EndocrinolMetab. 2011; 15:S44-9.

14. Bhat RA, Laway BA, Zargar AH (2010) Prevalence of metabolic syndrome in Kashmir valley of Indian subcontinent. Indian J Med Sci 64: 259-264.

15. Indulekha, K., Surendar, J. and Mohan, V. 2011. High sensitivity C-reactive protein, tumor necrosis factor- $\alpha$, interleukin-6, and vascular cell adhesion molecule-1 levels in Asian Indians with metabolic syndrome and insulin resistance (CURES-105). Journal of diabetes science and technology, 5 (4), pp. 982--988.

16. Hanley A, Williams K, Festa A, Wagenknecht L, D'Agostino R, Haffner S. Liver Markers and Development of the Metabolic Syndrome The Insulin Resistance Atherosclerosis Study. Diabetes. 2005; 54 (11): 3140--3147.

17. Elizondo-Montemayor L, Ugalde-Casas PA, Lam-Franco L, Bustamante-CareagaH,Serrano-Gonzalez M, Gutiérrez NG, Martinez U. Association of ALT and the metabolic syndrome among Mexican children. Obes Res Clin Pract.2014 Jan-Feb; 8(1):e79-87.

18. Park H, Han J, Choi K, Kim S. Relation between elevated serum alanine aminotransferase and metabolic syndrome in Korean adolescents. The American journal of clinical nutrition. 2005; 82 (5): 1046--1051. 
19. Qiang Lu, Xiaoli Liu, Shuhua Liu, ChangshunXie, Yali Liu and Chunming Ma (2012). The Relationship Between AST/ALT Ratio and Metabolic Syndrome in Han Young Adults - AST/ALT Ratio and Metabolic Syndrome, Recent Advances in Cardiovascular Risk Factors, Prof. MehnazAtiq (Ed.), ISBN: 978-953-51-03219, InTech, DOI: 10.5772/31633.

20. Chen Z, Chen L, Dai H, Chen J, Fang L. Relationship between alanine aminotransferase levels and metabolic syndrome in nonalcoholic fatty liver disease. Journal of Zhejiang University Science B. 2008; 9 (8): 616--622.

21. Chen P H, Chen J D, Lin Y C. A better parameter in predicting insulin resistance: obesity plus elevated alanine aminotransferase. World J Gastroenterol 2009, 15:5598-5603.

22. Kawamoto R, Kohara K, Kusunoki T, Tabara Y, Abe M, Miki T, Others. Alanine aminotransferase/aspartate aminotransferase ratio is the best surrogate marker for insulin resistance in nonobese Japanese adults. CardiovascDiabetol. 2012; 11: 117.

23. Gao F, Pan JM, Hou XH, Fang QC, Lu HJ, Tang JL, et al. Liver enzymes concentrations are closely related to prediabetes: Findings of the Shanghai Diabetes Study II (SHDS II)Biomed Environ Sci. 2012;25:30-7.

24. Esteghamati A, Jamali A, Khalilzadeh O, Noshad S, Khalili M, Zandieh A. et al., Metabolic syndrome is linked to a mild elevation in liver aminotransferases in diabetic patients with undetectable non-alcoholic fatty liver disease by ultrasound. DiabetolMetabSyndr 2010; 2:65. 